\title{
Factorization and subtraction
}

\section{Lorenzo Magnea*, Ezio Maina, Paolo Torrielli, Sandro Uccirati}

University of Torino and INFN, Sezione di Torino

E-mail: lorenzo.magnea@unito.it

We explore the connection between the factorisation of virtual corrections to multi-particle massless gauge theory amplitudes and the problem of subtraction at NNLO and beyond. Taking inspiration from virtual factorisation, we provide a set of definitions for local soft and collinear counterterms, expressed in terms of matrix elements of operators involving fields and Wilson lines, and valid to all orders in perturbation theory. We hope that the connection between factorisation and subtraction will help in the construction of minimal, stable, and efficient subtraction algorithms, taking maximal advantage of existing analytic information.

13th International Symposium on Radiative Corrections 24-29 September, 2017

St. Gilgen, Austria

\section{* Speaker}




\section{Introduction}

The infrared structure of virtual corrections to perturbative gauge theory amplitudes is very well understood [1, 2, 3, 4, 5, 6, 7]: soft and collinear singularities associated with on-shell configurations of loop momenta are known to factorise from the hard scattering, and they follow a pattern of exponentiation dictated by a small set of soft and collinear anomalous dimensions, which (in the massless case) are known to three loops [8]. Similarly, the factorisation properties of real radiation amplitudes, in the limits when one or more external partons become soft, or collinear to each other, are understood in considerable generality $[9,10,11]$, and all the splitting kernels (at the amplitude level) necessary for next-to-next-to-leading order (NNLO) calculations have been computed $[11,12,13,14]$.

Notwithstanding these early results, the problem of constructing general and efficient algorithms for the calculation of infrared-safe observables in hadronic scattering processes beyond NLO has proven to be very challenging. After pioneering results on jet production in electron-positron annihilation $[15,16]$, several approaches have been developed to tackle the problem for hadron scattering; some have already been successfully deployed to selected processes at NNLO, while some are in different stages of development: several talks at this conference have discussed recent developments of the various proposed methods and their applications [17, 18, 19, 20, 21, 22, 23, 24]. To give an extremely concise summary of the state of the art, we note that the first fully hadronic process to be studied at NNLO at the level of fully differential distributions was the production of top-antitop pairs [25, 26], a seminal result achieved using a predominantly numerical subtraction scheme; processes with one final-state jet, or with electroweak final states, have subsequently been tamed using phase-space slicing methods such as N-Jettiness subtraction [27, 28, 29], or $q_{T}$ subtraction [30, 31, 32]; very recently, the first complete results for dijet production (in the planar limit) have been presented [33], using Antenna subtraction. Other methods have been applied using process-specific approximations [34], or to processes with electroweak initial states [35, 36, 37], while yet other methods are at the design stage [38], or undergoing preliminary testing [39]. Finally, it is worth pointing out that limited extensions to $\mathrm{N}^{3} \mathrm{LO}$ [40] are being attempted.

All the methods devised and implemented so far are very demanding, either in terms of the necessary numerical calculations, or because of the intricacy of the analytical integrations to be performed, or both, underscoring the difficulty of the problem. At the same time, the massive amounts of data being delivered by LHC, and the increasing requirements on the precision of theoretical predictions for a widening array of processes, point to the necessity of preparing for much more intricate applications. In the coming years, differential calculations for multi-jet final states at NNLO, and for selected observables at $\mathrm{N}^{3} \mathrm{LO}$, are likely to become both feasible and relevant. In light of current results, this kind of generalisation will likely require a massive optimisation and streamlining of current subtraction methods, and possibly the development of entirely new ones.

In the present contribution, we would like to present some preliminary results in this direction. Specifically, we explore the relationship between the factorisation of IR divergences in virtual corrections to scattering amplitudes, and the local counterterms required for infrared subtraction. This investigation has a two-fold purpose. On the one hand, as we will see, it provides a 'shopping list' of gauge-invariant operator matrix elements which yield soft and collinear counterterms to any order in perturbation theory; in principle, this allows to decouple the identification of the coun- 
terterms from the calculation of specific scattering amplitudes, in contrast to the methods followed so far at the two-loop level (see, for example, [41, 42]). On the other hand, by tracing an explicit connection between virtual corrections and real radiation, one may hope that the remarkable simplifications inherent in virtual factorisation may be reflected in the properties of the counterterms: for example, high orders in the virtual soft and jet functions are tied to low orders by exponentiation, and furthermore it is known a priori that entire classes of contributions to soft anomalous dimensions vanish to all orders in perturbation theory $[4,7]$. We will begin, in Section 2, by very briefly reviewing what is known about the IR factorisation of fixed-angle multi-parton scattering amplitudes; we will then present a basic outline of the subtraction problem in Section 3, focusing for simplicity on the NLO case; in Sections 4 and 5, we present and motivate our general ansatz for soft and collinear subtraction counterterms to all orders; in Section 6, as an example, we show how our definitions combine to reconstruct the well-understood structure of final-state IR subtraction at NLO; finally, we give a brief perspective in Section 7.

\section{Infrared structure of virtual corrections}

Massless multi-particle gauge theory scattering amplitudes are affected by soft and collinear divergences beyond leading order in perturbation theory. Divergences arise from long-distance contributions to virtual corrections, and therefore enjoy a degree of universality, being largely independent from the details of the hard scattering. Divergent contributions can therefore be expressed in terms of universal, gauge-invariant operator matrix elements, multiplying a finite hard remainder. The final result can be derived using diagrammatic techniques [43], or effective field theory methods [44], and can be expressed in terms of an all-order factorisation theorem. Writing the $n$-particle scattering amplitude as $A^{a_{1} \ldots a_{n}}$, where the colour indices $a_{i}$ can belong to any representation of the gauge group, the first step is to choose a basis in the space of available color structures for the given process, by picking a suitable set of color tensors $c_{K}^{a_{1} \ldots a_{n}}$. The scattering amplitude can then be expressed in terms of its components $A_{K}$ in this basis. These 'partial amplitudes' obey the factorisation

$$
A_{K}\left(p_{i} / \mu\right)=\prod_{i=1}^{n}\left[J_{i}\left(\frac{\left(p_{i} \cdot n_{i}\right)^{2}}{n_{i}^{2} \mu^{2}}\right) / \mathscr{J}_{i}\left(\frac{\left(\beta_{i} \cdot n_{i}\right)^{2}}{n_{i}^{2}}\right)\right] \mathscr{S}_{K}^{L}\left(\beta_{i} \cdot \beta_{j}\right) H_{L}\left(\frac{p_{i} \cdot p_{j}}{\mu^{2}}, \frac{\left(p_{i} \cdot n_{i}\right)^{2}}{n_{i}^{2} \mu^{2}}\right),
$$

where for simplicity we have suppressed the dependence on the renormalised coupling $\alpha_{s}\left(\mu^{2}\right)$ and on the dimensional regularisation parameter $\varepsilon$, and where $p_{i}, i=1, \ldots, n$, are the external momenta, while $\beta_{i}$ are the corresponding dimensionless four-velocities, obtained by rescaling the momenta by a common hard scale, for example setting $p_{i}=\mu \beta_{i}$.

The 'jet' functions $J_{i}$ in Eq. (2.1) collect all collinear singularities associated with virtual quanta emitted in the direction of particle $i$; they depend on the particle spin, but they are 'colour singlet' quantities, in the sense that they do not affect the color of the external particle; they can be defined as operator matrix elements involving Wilson lines. For example, in the case of an outgoing quark, we can use

$$
\bar{u}(p) J\left(\frac{(p \cdot n)^{2}}{n^{2} \mu^{2}}\right)=\left\langle p\left|\bar{\psi}(0) \Phi_{n}(0, \infty)\right| 0\right\rangle
$$


where $\Phi_{n}$ is a semi-infinite Wilson line in an arbitrary direction $n_{i}^{\mu}$, with $n^{2} \neq 0$, according to the definition

$$
\Phi_{n}\left(\lambda_{2}, \lambda_{1}\right) \equiv \mathscr{P} \exp \left[\mathrm{ig} \int_{\lambda_{1}}^{\lambda_{2}} d \lambda n \cdot A(\lambda n)\right]
$$

The vectors $n_{i}$ play the role of 'factorisation vectors', and they ensure gauge invariance of the operator matrix element defining $J_{i}$. The specific functional dependence of $J_{i}$ on its argument is dictated by the masslessness of the external particle, $p_{i}^{2}=0$, and by the rescaling invariance of the semi-infinite Wilson line under $n_{i}^{\mu} \rightarrow \kappa_{i} n_{i}^{\mu}$, with $\kappa_{i}$ a constant.

The colour-singlet nature of the jet functions $J_{i}$ follows from collinear power counting at the level of Feynman graphs, and greatly simplifies the structure of the factorisation. Soft singularities, on the other hand, are not color diagonal, and therefore they are organised in a matrix, $\mathscr{S}_{L K}$, which is purely eikonal. Indeed, since soft gluons have long wavelength, they do not resolve the details of the hard interaction nor the internal structure of the jets, and therefore they couple effectively to Wilson lines in the colour representations of the corresponding hard external partons, and oriented along their four-velocities $\beta_{i}$. In a chosen basis of independent tensors $c_{K}$ in colour space, one defines

$$
\left(c_{L}\right)^{\left\{a_{k}\right\}} \mathscr{S}_{K}^{L}\left(\beta_{i} \cdot \beta_{j}\right)=\left\langle 0\left|\prod_{k=1}^{n}\left[\Phi_{\beta_{k}}(\infty, 0)^{a_{k} b_{k}}\right]\right| 0\right\rangle\left(c_{K}\right)_{\left\{b_{k}\right\}} .
$$

Notice that the dependence of the soft matrix on the four-velocities $\beta_{i}$ is severely constrained by the rescaling invariance of the semi-infinite Wilson line operators under $\beta_{i} \rightarrow \kappa_{i} \beta_{i}$ : in the massless case, only dependence on conformal invariant cross ratios of the form

$$
\rho_{i j k l} \equiv \frac{\beta_{i} \cdot \beta_{j} \beta_{k} \cdot \beta_{l}}{\beta_{i} \cdot \beta_{l} \beta_{j} \cdot \beta_{k}}
$$

would be allowed, except for the fact that the rescaling invariance of the correlator in Eq. (2.4) is broken by collinear poles, leading to an explicit but highly constrained dependence on $\beta_{i} \cdot \beta_{j}$, proportional to the cusp anomalous dimension $[4,5,6,7]$.

The final ingredients of the factorisation formula Eq. (2.1) are the soft approximations of the jet functions $J_{i}$, sometimes called call 'eikonal jets'. They are defined by

$$
\mathscr{J}_{i}\left(\frac{\left(\beta_{i} \cdot n_{i}\right)^{2}}{n_{i}^{2}}\right)=\left\langle 0\left|\Phi_{\beta_{i}}(\infty, 0) \Phi_{n_{i}}(0, \infty)\right| 0\right\rangle .
$$

Introducing eikonal jets is necessary in order to avoid double counting of gluons that are both soft and collinear to one of the hard external partons: such gluons appear both in the jet functions $J_{i}$ and in the soft matrix $\mathscr{S}$. It is however simple to subtract this double counting: one just needs to divide each jet $J_{i}$ by its own soft approximation $\mathscr{J}_{i}$, as done in Eq. (2.1). Notice once again the argument of the eikonal jet functions $\mathscr{J}_{i}$ : we allow only for homogeneous dependence on the (non-light-like) vectors $n_{i}$, while non-homogeneous dependence on the light-like vectors $\beta_{i}$ is allowed, as was the case for the soft matrix $\mathscr{S}$.

The content of the factorisation theorem in Eq. (2.1) is that all soft and collinear divergences in the amplitude, to all orders in perturbation theory, are generated by the universal soft and jet 
functions: the vector of hard functions $H_{K}$ is then a matching coefficient, collecting all finite remainders, and it is finite as $\varepsilon \rightarrow 0$. The theorem is powerful because, like all factorisations, it implies the existence of evolution equations, which can be solved to construct a resummation (exponentiation) of infrared poles. To briefly illustrate this fact, we note that Eq. (2.1) can be rewritten by collecting soft and collinear factors in a form reminiscent of ultraviolet renormalisation, as [5, 6]

$$
\mathscr{A}\left(\frac{p_{i}}{\mu}\right)=\mathscr{Z}\left(\frac{p_{i}}{\mu}\right) \mathscr{H}\left(\frac{p_{i}}{\mu}\right)
$$

where the cancellation of the dependence on the factorisation vectors $n_{i}$ between the various factors comprising $\mathscr{Z}$ and $\mathscr{H}$ has already been implemented, the scattering amplitude $\mathscr{A}$ is a vector in color space, and $\mathscr{Z}$ is a matrix encoding all infrared divergences, acting upon the finite vector $\mathscr{H}$. The infrared operator $\mathscr{Z}$ obeys a renormalisation group equation which, in dimensional regularization [45] and for $d=4-2 \varepsilon>4$, has a very simple solution, expressed in terms of a soft anomalous dimension matrix $\Gamma$ as

$$
\mathscr{Z}\left(\frac{p_{i}}{\mu}, \alpha_{s}\left(\mu^{2}\right)\right)=\mathscr{P} \exp \left[\frac{1}{2} \int_{0}^{\mu^{2}} \frac{d \lambda^{2}}{\lambda^{2}} \Gamma\left(\frac{p_{i}}{\lambda}, \alpha_{s}\left(\lambda^{2}\right)\right)\right] .
$$

It is clear from this discussion that the matrix $\Gamma$ is the key ingredient for the solution of the perturbative IR problem: it is currently known at three loops for massless particles [8]. In the context of the subtraction problem, we note that the exponentiation in Eq. (2.8), and the highly non-trivial form of the matrix $\Gamma[4,7,8]$, imply a number of connections between virtual IR poles at different orders, as well as significant constraints on their structure. These connections and constraints must at some level be reflected by the real radiation counterterms: elucidating this relationship is one of the goals of our approach.

\section{Infrared subtraction: an outline}

In order to begin our discussion, let us start with a very concise summary of the subtraction procedure at lowest non-trivial order. For the sake of simplicity, we will focus on final state radiation only. We consider a generic infrared-safe observable $O$, which receives its leading order contribution from a final state with $n$ partons. For such an observable, NLO distributions are in principle computed as

$$
\langle O\rangle_{\mathrm{NLO}}=\lim _{d \rightarrow 4}\left\{\int d \Phi_{n}\left[B_{n}+V_{n}\right] O_{n}+\int d \Phi_{n+1} R_{n+1} O_{n+1}\right\},
$$

where $B_{n}$ is the Born-level squared amplitude, $V_{n}$ the corresponding one-loop corrections, and $R_{n+1}$ is the single-real-radiation tree-level squared amplitude. The observable $O$ admits explicit expressions $O_{m}$ in the $m$-particle phase space, and IR safety requires that $O_{n+1} \rightarrow O_{n}$ in all degenerate limits where one particle becomes unresolved, becoming soft or collinear to another one. The practical problem in evaluating Eq. (3.1) is that the r.h.s. of Eq. (3.1) must in general be evaluated numerically (due to the complexity of the typical observable $O$ and of the relevant matrix elements), while the explicit poles in the first term must be cancelled against singularities arising in the phase-space integration of the second term. The idea of subtraction is to introduce a set of 
local counterterm functions $K_{m}$, with the property that they reproduce the singular behaviour of the real-radiation squared matrix element $R_{n+1}$ in all unresolved limits, while at the same time being simple enough to be analytically integrated over the single-unresolved-particle phase space. One then defines

$$
\langle O\rangle_{\mathrm{ct}}=\int d \Phi_{n} d \hat{\Phi}_{1} K_{n+1} O_{n},
$$

and then proceeds to compute analytically the integral

$$
I_{n}=\int d \hat{\Phi}_{1} K_{n+1}
$$

Eq. (3.1) can now be rewritten identically as

$$
\langle O\rangle_{\mathrm{NLO}}=\int d \Phi_{n}\left[B_{n}^{(4)}+\left(V_{n}+I_{n}\right)^{(4)}\right] O_{n}+\int d \Phi_{n}\left[\int d \Phi_{1}^{(4)} R_{n+1}^{(4)} O_{n+1}-\int d \hat{\Phi}_{1}^{(4)} K_{n+1}^{(4)} O_{n}\right] .
$$

By the standard theorems concerning the cancellation of IR singularities, the two terms in Eq. (3.4) are now separately finite and can be evaluated numerically in $d=4$.

Clearly, at higher orders this relatively straightforward procedure becomes much more intricate, due to the presence of a large number of overlapping singular regions. Furthermore, this sketchy formal treatment neglects the technical difficulties associated with the need to construct precise and efficient phase space mappings relating the radiative and the Born configurations: our viewpoint on these issues is discussed in [18]. In what follows, we focus on the general structure of the counterterm functions $K_{m}$. As we will see below, the fact that the structure of virtual poles is known to all orders in terms of gauge invariant operator matrix elements suggests a completely general definition of soft and collinear counterterms to any order; furthermore, the structure of the virtual factorisation also dictates a pattern of cancellation of overlapping singularities, in principle allowing for an automatic construction of the subtraction procedure at NNLO and beyond.

\section{Local soft counterterms}

Let us begin by focusing on purely soft singular configurations. Real soft radiation at leading power is described by replacing hard radiating particles with Wilson lines, so one is naturally led to consider the matrix elements for the radiation of $m$ soft partons from $n$ (outgoing) Wilson lines,

$$
\begin{aligned}
\mathscr{S}_{\lambda_{1} \ldots \lambda_{m}}\left(k_{1}, \ldots, k_{m} ; \beta_{i}\right) & \equiv\left\langle k_{1}, \lambda_{1} ; \ldots ; k_{m}, \lambda_{m}\left|\prod_{i=1}^{n} \Phi_{\beta_{i}}(\infty, 0)\right| 0\right\rangle \\
& \equiv g^{m} \varepsilon_{\lambda_{1}}^{* \mu_{1}}\left(k_{1}\right) \ldots \varepsilon_{\lambda_{m}}^{* \mu_{m}}\left(k_{m}\right) J_{\mu_{1} \ldots \mu_{m}}^{\mathscr{S}}\left(k_{1}, \ldots, k_{m} ; \beta_{i}\right) \\
& \equiv g^{m} \sum_{p=0}^{\infty}\left(\frac{\alpha_{s}}{\pi}\right)^{p} \mathscr{S}_{\lambda_{1} \ldots \lambda_{m}}^{(p)}\left(k_{1}, \ldots, k_{m} ; \beta_{i}\right),
\end{aligned}
$$

where in the second line we have extracted polarisation vectors ${ }^{1}$ and an overall power of the coupling, in order to define a multi-gluon soft emission current $J_{\mu_{1} \ldots \mu_{m}}^{\mathscr{S}}$, while in the third line we have defined the perturbative coefficients of the loop expansion of our matrix element.

\footnotetext{
${ }^{1}$ Here we consider only radiated gluons, but it is straightforward to generalise the definition to quarks.
} 
We note that existing finite-order calculations and all-order arguments are consistent with the soft factorisation of the radiative matrix element

$$
\mathscr{A}_{\lambda_{1} \ldots \lambda_{m}}\left(k_{1}, \ldots, k_{m} ; p_{i}\right) \simeq \mathscr{S}_{\lambda_{1} \ldots \lambda_{m}}\left(k_{1}, \ldots, k_{m} ; \beta_{i}\right) \mathscr{H}\left(p_{i}\right)
$$

with corrections that are finite in dimensional regularisation and integrable in the soft-gluon phase space. Squaring the matrix element and (optionally) summing over polarisations one can then write

$$
\sum_{\lambda_{1} \ldots \lambda_{m}}\left|\mathscr{A}_{\lambda_{1} \ldots \lambda_{m}}\left(k_{1}, \ldots, k_{m} ; p_{i}\right)\right|^{2} \simeq S_{m}\left(k_{1}, \ldots, k_{m} ; \beta_{i}\right)\left|\mathscr{H}\left(p_{i}\right)\right|^{2}
$$

which defines the cross-section-level radiative soft function

$$
\begin{aligned}
S_{m}\left(k_{1}, \ldots, k_{m} ; \beta_{i}\right) & \equiv \sum_{\lambda_{1} \ldots \lambda_{m}}\left\langle 0\left|\prod_{i=1}^{n} \Phi_{\beta_{i}}(0, \infty)\right| k_{1}, \lambda_{1} ; \ldots ; k_{m}, \lambda_{m}\right\rangle\left\langle k_{1}, \lambda_{1} ; \ldots ; k_{m}, \lambda_{m}\left|\prod_{i=1}^{n} \Phi_{\beta_{i}}(\infty, 0)\right| 0\right\rangle \\
& \equiv\left(4 \pi \alpha_{s}\right)^{m} \sum_{p=0}^{\infty}\left(\frac{\alpha_{s}}{\pi}\right)^{p} S_{m}^{(p)}\left(k_{1}, \ldots, k_{m} ; \beta_{i}\right)
\end{aligned}
$$

It is clear that the perturbative coefficients $S_{m}^{(p)}\left(k_{1}, \ldots, k_{m} ; \beta_{i}\right)$ are ideally suited to act as minimal local counterterms for the soft singular regions of phase space, to any order in perturbation theory. Indeed, summing over the number of particles in the final state and integrating over their phase space, by completeness we find

$$
\sum_{m=0}^{\infty} \int d \Phi_{m}\left(k_{j}\right) S_{m}\left(k_{1}, \ldots, k_{m} ; \beta_{i}\right)=\left\langle 0\left|\prod_{i=1}^{n} \Phi_{\beta_{i}}(0, \infty) \prod_{i=1}^{n} \Phi_{\beta_{i}}(\infty, 0)\right| 0\right\rangle .
$$

The right-hand side is effectively a total eikonal cross section, and thus finite order by order by the general theorems concerning the cancellation of IR divergences. The $m=0$ term gives the purely virtual contribution, and one easily verifies diagrammatically that real radiation corrections construct order by order the familiar pattern of cancellations.

In order to illustrate the results, and to make contact with earlier work, in particular [11, 14], consider first the tree-level radiative amplitude with a single soft gluon. Eq. (4.2) in this case reduces to

$$
\mathscr{A}_{\lambda}^{(0)}\left(k, p_{i}\right)=g \varepsilon_{\lambda}^{*}(k) \cdot J_{(0)}^{\mathscr{S}}\left(k, \beta_{i}\right) \mathscr{H}^{(0)}\left(p_{i}\right)+\mathscr{O}\left(k^{0}\right) .
$$

The definition in Eq. (4.1) at this level reduces to

$$
g \varepsilon_{\lambda}^{*}(k) \cdot J_{(0)}^{\mathscr{S}}\left(k, \beta_{i}\right)=\left.\left\langle k, \lambda\left|\prod_{i=1}^{n} \Phi_{\beta_{i}}(\infty, 0)\right| 0\right\rangle\right|_{\text {treelevel }},
$$

which immediately yields the well-known result

$$
J_{(0)}^{\mathscr{S}, \mu}\left(k, \beta_{i}\right)=\sum_{i=1}^{n} \frac{\beta_{i}^{\mu}}{\beta_{i} \cdot k} \mathbf{T}_{i}
$$


Squaring the tree amplitude in Eq. (4.6) and summing over soft-gluon polarisations yields the factorisation of the standard eikonal prefactor

$$
\begin{aligned}
\sum_{\lambda}\left|\mathscr{A}_{\lambda}^{(0)}\left(k, p_{i}\right)\right|^{2} & \simeq-4 \pi \alpha_{s} \sum_{i, j=1}^{n} \frac{\beta_{i} \cdot \beta_{j}}{\beta_{i} \cdot k \beta_{j} \cdot k} \mathscr{A}^{(0) \dagger}\left(p_{i}\right) \mathbf{T}_{i} \cdot \mathbf{T}_{j} \mathscr{A}^{(0)}\left(p_{i}\right) \\
& =S_{1}^{(0)}\left(k ; \beta_{i}\right)\left|\mathscr{H}^{(0)}\left(p_{i}\right)\right|^{2}
\end{aligned}
$$

In the second line of Eq. (4.9) we have adopted a schematic operator notation, defined by the first line: the one-gluon tree-level radiative soft function is a color operator acting on the colourcorrelated Born squared matrix element, which at tree level is finite and coincides with the hard part. As one might expect, the two-gluon soft current can similarly be computed, recovering the results of [11], and multiple soft gluon currents follow the same pattern.

The picture gets a bit more interesting at one loop. Considering as an example single-gluon emission, one can compare the factorisation in Eq. (4.2) with the one proposed in Ref. [14]. They read

$$
\mathscr{A}_{\lambda}\left(k ; p_{i}\right) \simeq \mathscr{S}_{\lambda}\left(k ; \beta_{i}\right) \mathscr{H}\left(p_{i}\right), \quad \mathscr{A}_{\lambda}\left(k ; p_{i}\right) \simeq g \varepsilon_{\lambda}^{*}(k) \cdot J_{C G}\left(k, \beta_{i}\right) \mathscr{A}\left(p_{i}\right),
$$

respectively, where we denoted with $J_{C G}$ the Catani-Grazzini soft-gluon current. Intuitively, the difference is that virtual IR divergences are still contained in the non-radiative matrix element $\mathscr{A}$ in the Catani-Grazzini factorisation, whereas the hard factor $\mathscr{H}$ is finite. It is however easy to map the two expressions in Eq. (4.10): expanding the one-loop amplitude in the first expression in Eq. (4.10) we find

$$
\mathscr{A}_{\lambda}^{(1)}\left(k ; p_{i}\right) \simeq \mathscr{S}_{\lambda}^{(0)}\left(k ; \beta_{i}\right) \mathscr{H}^{(1)}\left(p_{i}\right)+\mathscr{S}_{\lambda}^{(1)}\left(k ; \beta_{i}\right) \mathscr{H}^{(0)}\left(p_{i}\right) .
$$

We can then express the one loop non-radiative hard part in terms of the full amplitude using Eq. (4.2) for $m=0$, obtaining

$$
\mathscr{A}\left(p_{i}\right) \simeq \mathscr{S}\left(\beta_{i}\right) \mathscr{H}\left(p_{i}\right) \quad \longrightarrow \quad \mathscr{H}^{(1)}\left(p_{i}\right)=\mathscr{A}^{(1)}\left(p_{i}\right)-\mathscr{S}^{(1)}\left(\beta_{i}\right) \mathscr{A}^{(0)}\left(p_{i}\right),
$$

where for simplicity we reabsorbed the tree-level soft function, a pure color factor, into $\mathscr{H}^{(1)}$. Substituting this result in Eq. (4.10), and solving for the one-loop soft current, we find

$$
g^{3} \varepsilon_{\lambda}^{*}(k) \cdot J_{C G}^{(1)}\left(k, \beta_{i}\right) \mathscr{A}^{(0)}\left(p_{i}\right)=\left[\mathscr{S}_{\lambda}^{(1)}\left(k ; \beta_{i}\right)-\mathscr{S}_{\lambda}^{(0)}\left(k ; \beta_{i}\right) \mathscr{S}^{(1)}\left(\beta_{i}\right)\right] \mathscr{A}^{(0)}\left(p_{i}\right) .
$$

Working out the diagrammatics, it is not difficult to recover the full result of Ref. [14] for the oneloop current: in a sense, Eq. (4.13) provides a transparent interpretation of the cancellations that lead to the purely non-abelian structure of $J_{C G}^{(1)}$ first derived in [14]. It is not difficult to extend these structural arguments to either more gluons ore more loops, obtaining precise operator definitions of the corresponding soft currents: this can lead, for example, to a first-principle calculation of the two-loop soft-gluon current first computed in [41, 42], and to a generalisation of that result beyond the two-hard-parton case. 


\section{Local collinear counterterms}

Local collinear counterterms can be constructed with the same method, starting from the definition of virtual jets, and suitably allowing for final state radiation. More precisely, for example in the case of a final state quark jet, consider the matrix elements ${ }^{2}$

$$
\mathscr{J}_{m, s}^{\lambda_{j}}\left(k_{j} ; p, n\right) \equiv\left\langle p, s ; k_{j}, \lambda_{j}\left|\bar{\psi}(0) \Phi_{n}(0, \infty)\right| 0\right\rangle \equiv g^{m} \sum_{p=0}^{\infty}\left(\frac{\alpha_{s}}{\pi}\right)^{p} \mathscr{J}_{m, s, \lambda_{j}}^{(p)}\left(k_{j} ; p, n\right)
$$

where $s$ is the quark spin and $\lambda_{j}$ are the gluon polarisations. Note that, at this stage, spinors and polarisation vectors for final-state partons are included in the definitions of $\mathscr{J}$ and of its perturbative coefficients $\mathscr{J}^{(p)}$ : one could extract them, as done in the second line of Eq. (4.1), and define 'collinear currents' for the emission of a given number of collinear final-state partons. Because of the non-trivial momentum flow in the collinear limit, rather than simply squaring the radiative jet amplitude in Eq. (5.1) it is appropriate to introduce a shift in the complex conjugate amplitude and then perform a Fourier transform, defining cross-section-level radiative jets as

$$
\begin{aligned}
J_{m}^{s, \lambda_{j}}\left(k_{j} ; l, p, n\right) & \equiv \int d^{d} x \mathrm{e}^{\mathrm{i} l \cdot x}\left\langle 0\left|\Phi_{n}(\infty, x) \psi(x)\right| p, s ; k_{j}, \lambda_{j}\right\rangle\left\langle p, s ; k_{j}, \lambda_{j}\left|\bar{\psi}(0) \Phi_{n}(0, \infty)\right| 0\right\rangle \\
& \equiv\left(4 \pi \alpha_{s}\right)^{m} \sum_{p=0}^{\infty}\left(\frac{\alpha_{s}}{\pi}\right)^{p} J_{m, s, \lambda_{j}}^{(p)}\left(k_{j} ; l, p, n\right) .
\end{aligned}
$$

The perturbative coefficients of the radiative jets, defined in the second line of Eq. (5.2), are natural possible definitions of collinear counterterms. Indeed, applying again completeness, one finds that

$$
\begin{aligned}
\sum_{m=0}^{\infty} \int d \Phi_{m+1} & \left(p, k_{j}\right) \sum_{\left\{\lambda_{j}\right\}} J_{m, s, \lambda_{j}}\left(k_{j} ; l, p, n\right)= \\
= & \operatorname{Disc}\left[\int d^{d} x \mathrm{e}^{\mathrm{i} l \cdot x}\left\langle 0\left|\Phi_{n}(\infty, x) \psi(x) \bar{\psi}(0) \Phi_{n}(0, \infty)\right| 0\right\rangle\right] .
\end{aligned}
$$

The right-hand side of Eq. (5.3) is a generalised two-point function (closely related to the inclusive jet function defined in [49]) and it is manifestly finite, since it is fully inclusive in the final state. Cross-section-level eikonal jets can be defined analogously, as

$$
\begin{aligned}
J_{m, \lambda_{j}}^{\mathrm{E}}\left(k_{j} ; l, \beta, n\right) & \equiv \int d^{d} x \mathrm{e}^{\mathrm{i} l \cdot x}\left\langle 0\left|\Phi_{n}(\infty, x) \Phi_{\beta}(x, \infty)\right| k_{j}, \lambda_{j}\right\rangle\left\langle k_{j}, \lambda_{j}\left|\Phi_{\beta}(\infty, 0) \Phi_{n}(0, \infty)\right| 0\right\rangle \\
& \equiv\left(4 \pi \alpha_{s}\right)^{m} \sum_{p=0}^{\infty}\left(\frac{\alpha_{s}}{\pi}\right)^{p} J_{m, \lambda_{j}}^{\mathrm{E},(p)}\left(k_{j} ; l, \beta, n\right),
\end{aligned}
$$

where now the Fourier transform simply sets the total final state momentum of soft-collinear gluons to be $l^{\mu}$, and once again polarisation vectors have been included in the definition. Note that eikonal jets, as expected, do not depend on the spin of the hard emitting parton.

As a sanity check, one can compute the single-gluon radiative jet: at cross-section level, summing over polarisations, one should recover the (unpolarised) Altarelli-Parisi splitting function $P_{q q}$.

\footnotetext{
${ }^{2}$ The amplitude-level radiative jets defined in Eq. (5.1) are closely related (but not identical) to the ones introduced in Refs. [46, 47, 48].
} 
Indeed, a straightforward calculation yields

$$
\sum_{s, \lambda} J_{1}^{s, \lambda}(k ; l, p, n)=\frac{4 \pi \alpha_{s} C_{F}}{l^{2}}(2 \pi)^{d} \delta^{d}(l-p-k)\left[-l \gamma_{\mu} \not p \gamma^{\mu} l+\frac{1}{k \cdot n}(l n \not p p+\not p h l)\right],
$$

up to terms proportional to $n^{2}$. Introducing a Sudakov parametrisation

$$
p^{\mu}=z l^{\mu}+\mathscr{O}\left(l_{\perp}\right), \quad k^{\mu}=(1-z) l^{\mu}+\mathscr{O}\left(l_{\perp}\right), \quad n^{2}=0,
$$

and taking the collinear limit $l_{\perp} \rightarrow 0$ one easily finds

$$
\sum_{s, \lambda} J_{1}^{s, \lambda}(k ; l, p, n)=\frac{8 \pi \alpha_{s} C_{F}}{l^{2}}(2 \pi)^{d} \delta^{d}(l-p-k)\left[\frac{1+z^{2}}{1-z}-\varepsilon(1-z)+\mathscr{O}\left(l_{\perp}\right)\right] .
$$

Once again, a detailed mapping to the axial-gauge calculation of Ref. [11] is possible: diagrams involving gluon emission from the Wilson lines in our calculation reconstruct the non-trivial terms arising from the axial-gauge gluon propagator in [11].

We note that, unlike the situation for soft radiation, the collinear counterterms defined by Eq. (5.2) are not minimal: the total final state momentum $l^{\mu}$ is generic, and in principle the collinear limit must be taken at the end of the calculation. Furthermore, while the matrix elements are gaugeinvariant, they are $n$-dependent: at loop level, choosing $n^{2}=0$ from the outset would need to be done with care, since in this case there are spurious collinear divergences in loops associated with emissions from the Wilson line (see Ref. [47] for a discussion of this issue). In short, there is room for improvement in our definition of collinear counterterms, and a refinement is likely to be practically necessary when tackling high-order corrections.

\section{NLO subtraction: a sketch}

We conclude this brief discussion with an outline of how the framework we have introduced translates into a sequence of steps to construct a subtraction scheme. Here we will only describe very schematically what happens at NLO, a more detailed account will be found in [50]. The main idea, as discussed in the Introduction, is to start from the structure of IR divergences in the virtual contribution to the observable distribution. We consider then the non-radiative scattering amplitude at the one-loop level, which is given by

$$
\begin{aligned}
\mathscr{A}\left(p_{i}\right)=\mathscr{S}^{(0)}\left(\beta_{i}\right) \mathscr{H}^{(0)}\left(p_{i}\right)+\frac{\alpha_{s}}{\pi}\left[\mathscr{S}^{(1)}\left(\beta_{i}\right) \mathscr{H}^{(0)}\left(p_{i}\right)+\mathscr{S}^{(0)}\left(\beta_{i}\right) \mathscr{H}^{(1)}\left(p_{i}\right)\right. \\
\left.+\sum_{i}\left(\mathscr{J}^{(1)}\left(p_{i}\right)-\mathscr{J}_{E}^{(1)}\left(\beta_{i}\right)\right) \mathscr{S}^{(0)}\left(\beta_{i}\right) \mathscr{H}^{(0)}\left(p_{i}\right)\right]+\mathscr{O}\left(\alpha_{s}^{2}\right) .
\end{aligned}
$$

The virtual term in Eq. (3.1) is then

$$
\begin{aligned}
V_{n} \equiv 2 \operatorname{Re}\left[\mathscr{A}_{n}^{(0) *} \mathscr{A}_{n}^{(1)}\right]= & \frac{\alpha_{s}}{\pi} S_{0}^{(1)}\left(\beta_{i}\right)\left|\mathscr{H}_{n}^{(0)}\left(p_{i}\right)\right|^{2} \\
& +\frac{\alpha_{s}}{\pi} \sum_{i}\left(J_{0}^{(1)}\left(p_{i}\right)-J_{E, 0}^{(1)}\left(\beta_{i}\right)\right)\left|\mathscr{H}_{n}^{(0)}\left(p_{i}\right)\right|^{2}+\text { finite. }
\end{aligned}
$$


To identify the real-radiation counterterms we now just need to expand the completeness relations for soft and collinear functions to NLO. At this order, they imply simply that

$$
S_{0}^{(1)}\left(\beta_{i}\right)+4 \pi^{2} \int d \Phi_{2}^{(d)} S_{1}^{(0)}\left(k, \beta_{i}\right)=\text { finite }
$$

and

$$
J_{0}^{(1)}(l, p, n)+4 \pi^{2} \int d \Phi_{2}^{(d)} J_{1}^{(0)}(k ; l, p, n)=\text { finite },
$$

where we have summed over polarisations for simplicity. Matching to Eq. (6.1), this means that local soft and collinear counterterms can be constructed according to

$$
K_{n+1}^{\mathrm{soft}}=4 \pi \alpha_{s} S_{1}^{(0)}\left(k, \beta_{i}\right)\left|\mathscr{H}_{n}^{(0)}\left(p_{i}\right)\right|^{2},
$$

while

$$
K_{n+1}^{\text {coll. }}=4 \pi \alpha_{s} \sum_{i} J_{1}^{(0)}\left(k_{i} ; l, p_{i}, n_{i}\right)\left|\mathscr{M}_{n}^{(0)}\left(p_{1}, \ldots, p_{i-1}, l, p_{i+1}, \ldots, p_{n}\right)\right|^{2}
$$

These results precisely correspond to the well-known, standard ones, as can be seen from Eq. (4.9) and Eq. (5.7). To avoid double counting, one must still subtract from the collinear counterterm the soft-collinear limit, which emerges as expected from Eq. (5.4). Pursuing the same approach at higher orders, starting at NNLO, we expect to develop a systematic viewpoint, which should allow us to take advantage of the well-organised structure of virtual corrections to simplify the interplay of real radiation counterterms. Clearly, what we have given here is just a set of definitions and prescriptions: they have the advantage of being valid to all perturbative orders and being universal across multi-particle massless scattering amplitudes; on the other hand, to build an actual subtraction algorithm, one still needs to work through the details of phase-space parametrisations, momentum mappings, and, of course, the necessary integrations of counterterms over the unresolved phase spaces. Our approach to these essential practical problems has been outlined in [18].

\section{Perspectives}

We have outlined an approach to the subtraction problem which starts from the well-known factorisation of virtual correction to multi-parton scattering amplitudes, and we have derived a completely general set of definitions for local subtraction counterterms, valid to all orders and for any massless gauge theory amplitude. We hope this approach will help in simplifying the structure of subtraction and the analytic integration of counterterms at NNLO and beyond. Work is in progress to construct a concrete subtraction algorithm incorporating these ideas into a practical and efficient framework, applicable to relevant LHC processes.

\section{Acknowledgements}

The work of PT has received funding from the European Union Seventh Framework programme for research and innovation under the Marie Curie grant agreement N. 609402-2020 researchers: Train to Move (T2M). 


\section{References}

[1] S. Catani, Phys. Lett. B 427 (1998) 161 [hep-ph/9802439].

[2] G. F. Sterman and M. E. Tejeda-Yeomans, Phys. Lett. B 552 (2003) 48 [hep-ph/0210130].

[3] L. J. Dixon, L. Magnea and G. F. Sterman, JHEP 0808 (2008) 022 [arXiv:0805.3515 [hep-ph]].

[4] E. Gardi and L. Magnea, JHEP 0903 (2009) 079 [arXiv:0901.1091 [hep-ph]].

[5] E. Gardi and L. Magnea, Nuovo Cim. C 32N5-6 (2009) 137 [Frascati Phys. Ser. 50 (2010)] [arXiv:0908.3273 [hep-ph]].

[6] T. Becher and M. Neubert, Phys. Rev. Lett. 102 (2009) 162001 Erratum: [Phys. Rev. Lett. 111 (2013) no.19, 199905] [arXiv:0901.0722 [hep-ph]].

[7] T. Becher and M. Neubert, JHEP 0906 (2009) 081 Erratum: [JHEP 1311 (2013) 024] [arXiv:0903.1126 [hep-ph]].

[8] Ø. Almelid, C. Duhr and E. Gardi, Phys. Rev. Lett. 117 (2016) no.17, 172002 [arXiv:1507.00047 [hep-ph]].

[9] D. A. Kosower, Nucl. Phys. B 552 (1999) 319 [hep-ph/9901201].

[10] S. Catani and M. Grazzini, Phys. Lett. B 446 (1999) 143 [hep-ph/9810389].

[11] S. Catani and M. Grazzini, Nucl. Phys. B 570 (2000) 287 [hep-ph/9908523].

[12] J. M. Campbell and E. W. N. Glover, Nucl. Phys. B 527 (1998) 264 [hep-ph/9710255].

[13] Z. Bern, V. Del Duca, W. B. Kilgore and C. R. Schmidt, Phys. Rev. D 60 (1999) 116001 [hep-ph/9903516].

[14] S. Catani and M. Grazzini, Nucl. Phys. B 591 (2000) 435 [hep-ph/0007142].

[15] A. Gehrmann-De Ridder, T. Gehrmann, E. W. N. Glover and G. Heinrich, Phys. Rev. Lett. 100 (2008) 172001 [arXiv:0802.0813 [hep-ph]].

[16] S. Weinzierl, Phys. Rev. Lett. 101 (2008) 162001 [arXiv:0807.3241 [hep-ph]].

[17] M. Cacciari, these proceedings.

[18] P. Torrielli, these proceedings.

[19] R. Roentsch, these proceedings.

[20] A. Behring, these proceedings.

[21] S. Kallweit, these proceedings.

[22] A. Kardos, these proceedings.

[23] G. Rodrigo, these proceedings.

[24] T. Gehrmann, these proceedings.

[25] M. Czakon, D. Heymes and A. Mitov, Phys. Rev. Lett. 116 (2016) no.8, 082003 [arXiv:1511.00549 [hep-ph]].

[26] M. Czakon, P. Fiedler, D. Heymes and A. Mitov, JHEP 1605 (2016) 034 [arXiv:1601.05375 [hep-ph]].

[27] R. Boughezal, F. Caola, K. Melnikov, F. Petriello and M. Schulze, Phys. Rev. Lett. 115 (2015) no.8, 082003 [arXiv:1504.07922 [hep-ph]]. 
[28] R. Boughezal, J. M. Campbell, R. K. Ellis, C. Focke, W. T. Giele, X. Liu and F. Petriello, Phys. Rev. Lett. 116 (2016) no.15, 152001 [arXiv:1512.01291 [hep-ph]].

[29] R. Boughezal, J. M. Campbell, R. K. Ellis, C. Focke, W. Giele, X. Liu, F. Petriello and C. Williams, Eur. Phys. J. C 77 (2017) no.1, 7 [arXiv:1605.08011 [hep-ph]].

[30] M. Grazzini, S. Kallweit and M. Wiesemann, arXiv:1711.06631 [hep-ph].

[31] M. Grazzini, S. Kallweit, D. Rathlev and M. Wiesemann, JHEP 1705 (2017) 139 [arXiv:1703.09065 [hep-ph]].

[32] D. de Florian, M. Grazzini, C. Hanga, S. Kallweit, J. M. Lindert, P. Maierhöfer, J. Mazzitelli and D. Rathlev, JHEP 1609 (2016) 151 [arXiv:1606.09519 [hep-ph]].

[33] J. Currie, A. Gehrmann-De Ridder, T. Gehrmann, E. W. N. Glover, A. Huss and J. Pires, Phys. Rev. Lett. 119 (2017) no.15, 152001 doi:10.1103/PhysRevLett.119.152001 [arXiv:1705.10271 [hep-ph]].

[34] M. Cacciari, F. A. Dreyer, A. Karlberg, G. P. Salam and G. Zanderighi, Phys. Rev. Lett. 115 (2015) no.8, 082002 [arXiv:1506.02660 [hep-ph]].

[35] Z. Tulipánt, A. Kardos and G. Somogyi, Eur. Phys. J. C 77 (2017) no.11, 749 [arXiv:1708.04093 [hep-ph]].

[36] V. Del Duca, C. Duhr, A. Kardos, G. Somogyi and Z. Trócsányi, Phys. Rev. Lett. 117 (2016) no.15, 152004 [arXiv:1603.08927 [hep-ph]].

[37] V. Del Duca, C. Duhr, A. Kardos, G. Somogyi, Z. Szôr, Z. Trócsányi and Z. Tulipánt, Phys. Rev. D 94 (2016) no.7, 074019 [arXiv:1606.03453 [hep-ph]].

[38] G. F. R. Sborlini, F. Driencourt-Mangin and G. Rodrigo, JHEP 1610 (2016) 162 [arXiv:1608.01584 [hep-ph]].

[39] F. Caola, K. Melnikov and R. Röntsch, Eur. Phys. J. C 77 (2017) no.4, 248 [arXiv:1702.01352 [hep-ph]].

[40] F. Dulat, B. Mistlberger and A. Pelloni, arXiv:1710.03016 [hep-ph].

[41] C. Duhr and T. Gehrmann, Phys. Lett. B 727 (2013) 452 [arXiv:1309.4393 [hep-ph]].

[42] Y. Li and H. X. Zhu, JHEP 1311 (2013) 080 [arXiv:1309.4391 [hep-ph]].

[43] G. F. Sterman, In *Boulder 1995, QCD and beyond*327-406 [hep-ph/9606312].

[44] I. Feige and M. D. Schwartz, Phys. Rev. D 90 (2014) no.10, 105020 [arXiv:1403.6472 [hep-ph]].

[45] L. Magnea and G. F. Sterman, Phys. Rev. D 42 (1990) 4222.

[46] V. Del Duca, Nucl. Phys. B 345 (1990) 369.

[47] D. Bonocore, E. Laenen, L. Magnea, S. Melville, L. Vernazza and C. D. White, JHEP 1506 (2015) 008, [arXiv:1503.05156 [hep-ph]].

[48] D. Bonocore, E. Laenen, L. Magnea, L. Vernazza and C. D. White, JHEP 1612 (2016) 121, [arXiv:1610.06842 [hep-ph]].

[49] G. F. Sterman, Nucl. Phys. B 281 (1987) 310.

[50] L. Magnea, E. Maina, P. Torrielli e S. Uccirati, in preparation. 